\title{
Participación de mujeres en organizaciones sociales y redes familiares en contexto de violencia doméstica
}

\author{
*Cristina Arrom Suhurt ${ }^{1}$, María Auxiliadora Arrom Suhurt ${ }^{1}$, Mónica Ruoti ${ }^{1}$, \\ Maria del Pilar Fresco ${ }^{2}$, Carmen Marina Arrom Suhurt ${ }^{1}$, Marcos Capurro ${ }^{3}$ \\ ${ }^{1}$ Centro para el Desarrollo de la Investigación Científica (CEDIC-Díaz Gill). Paraguay \\ ${ }^{2}$ Cátedra de Psiquiatría de la Facultad de Ciencias Médicas. Universidad Nacional de Asunción. Paraguay \\ ${ }^{3}$ Centro de Adicciones Ministerio de Salud Pública y Bienestar Social. Paraguay
}

Cómo referenciar este artículo/ How to reference this article:
Arrom Suhurt C, Arrom Suhurt MA, Ruoti M, Fresco MP, Arrom Suhurt CM, Capurro $M$. Participación de mujeres en organizaciones sociales y redes familiares en contexto de violencia doméstica. Mem. Inst. Investig. Cienc. Salud. 2018; 16(1): 6-13

\section{R E S U M E N}

El aislamiento social de mujeres víctimas de violencia doméstica constituye un factor de riesgo agravante y perpetuador del fenómeno para la población afectada. El objetivo del trabajo fue vincular mujeres embarazadas víctimas de violencia doméstica con la participación en organizaciones sociales y redes familiares. Estudio retrospectivo, de fuentes secundarias con muestreo consecutivo de mujeres que acudieron a hospitales públicos de Alto Paraná, Paraguay. Se aplicó la Escala de Gelles y Straus y un cuestionario sobre participación en organizaciones sociales y contacto con familiares. El $70,1 \%(n=733)$ de las 1046 mujeres fue víctima de violencia psicológica; $23,9 \%(n=250)$ de violencia física; $7,5 \%$ $(n=78)$ de abuso sexual y 7,9\% $(n=83)$ de violencia económica. La participación en organizaciones fue reportada como sigue: $41 \%(n=429)$ en grupo de iglesia, $12,8 \%$ $(n=134)$ en cooperadora escolar, $16,3 \%(n=171)$ en organizaciones vecinales; $5,5 \%$ $(n=58)$ en clubes deportivos, $4,1 \%(n=43)$ clubes de madres, $3,4 \%(n=36)$ organizaciones de mujeres y $2,6 \%(n=27)$ en organizaciones sindicales. El hecho de ser víctima de violencia psicológica se asoció con la no participación en cooperadoras escolares $(p<0,045)$ $y$ al no frecuentar amigas $(p=0,020)$. Tanto ser víctima de abuso sexual $(p<0,004)$ como ser víctima de violencia económica $(0,028)$ se asociaron con no frecuentar a los padres. La población de estudio además de reunir características sociales de vulnerabilidad, es víctima de violencia doméstica asociada a su falta de participación en organizaciones sociales y redes familiares.

Palabras clave: participación social-redes, familiares-violencia, doméstica-embarazadas.

\section{Participation of women in social organizations and family networks in the context of domestic violence}

\section{A B S T R A C T}

Social isolation of women victims of domestic violence constitutes an aggravating and perpetuating risk factor for the phenomenon of the affected population. The objective of the study was to relate pregnant women victims of domestic violence with participation in social organizations and family networks. Retrospective study of secondary sources with consecutive sampling of women who attended public hospitals in Alto Paraná, Paraguay. Gelles and Straus Scale and a questionnaire about participation in social organizations and contact with family members were applied. Of the 1046 women, $70.1 \%(n=733)$ was victim of psychological violence; $23.9 \%(n=250)$ of physical violence; $7.5 \%(n=78)$ of sexual abuse and $7.9 \%(n=83)$ of economic violence. Participation in organizations was reported as follows: $41 \%(n=429)$ in church group, $12.8 \%(n=134)$ in school cooperative, $16.3 \%(n=171)$ in neighborhood organizations; $5.5 \%(n=58)$ in sports clubs, $4.1 \%(n=43)$ mothers' clubs, $3.4 \%(n=36)$ women's organizations and $2.6 \%(n=$ 
$27)$ in unions. The fact of being a victim of psychological violence was associated with the non-participation in school associations $(p<0.045)$ and not frequenting friends $(p=0.020)$. Both being a victim of sexual abuse $(p<0.004)$ and being a victim of economic violence (0.028) were associated with not frequenting the parents. The study population, in addition to gathering social characteristics of vulnerability, is a victim of domestic violence associated with their lack of participation in social organizations and family networks.

Keywords: social participation-family, networks-domestic, violence-pregnant women.

\section{INTRODUCCION}

La violencia contra la mujer, especialmente aquella ejercida por su pareja, así como la violencia sexual, constituyen un grave problema de salud pública y una violación de los derechos humanos. En todo el mundo se ha calculado que el $35 \%$ de las mujeres ha sufrido violencia de pareja o violencia sexual por terceros en algún momento de la vida ${ }^{(1-3)}$.

El daño causado por la violencia puede durar toda la vida, afectando a varias generaciones y con graves consecuencias para la salud, educación, empleo, bienestar económico de las personas afectadas, la familia y la sociedad ${ }^{(3)}$. Las consecuencias inmediatas y a largo plazo sobre diferentes formas de violencia incluyen lesiones físicas, embarazos no deseados, abortos, complicaciones ginecológicas, infecciones de transmisión sexual, trastorno de estrés post traumático y depresión, también comportamientos de alto riesgo como el tabaquismo, consumo perjudicial de alcohol y drogas, comportamiento sexual arriesgado (3). Además, empobrece a las mujeres y sus familias, reduce la producción económica y los recursos de los servicios públicos ${ }^{(3,4)}$.

La violencia infligida por la pareja trae aparejada, además, un grave impacto en el bienestar psíquico y social en las familias porque tiene efectos negativos en el desempeño del rol paterno y materno en la crianza de hijos e hijas y en logros educativos y laborales ${ }^{(3)}$. "Una mamá deprimida no solo padece la depresión, sino que no puede ser mamá"(4), es decir, carece de la empatía que necesita para registrar las necesidades emocionales de su hijo $^{(5)}$.

La Organización Mundial de la Salud (OMS) en un estudio sobre salud y violencia doméstica contra la mujer en 24.000 mujeres entre 15 y 49 años de edad de zonas urbanas y rurales de 10 países, encontró que entre $1 \%$ y $21 \%$ de las mujeres habían sufrido abuso sexual antes de los 15 años, entre $13 \%$ y $61 \%$ notificaron maltrato físico por parte de la pareja; entre $6 \%$ y $59 \%$ violencia sexual por parte de la pareja en algún momento de la vida y entre $0,3 \%$ y $11,5 \%$ violencia sexual infligida por otra persona ${ }^{(3)}$. En distintas partes del mundo se ha investigado sobre violencia doméstica en población de mujeres en situación de embarazo y se ha revelado una prevalencia de violencia física entre el $1 \%$ y $40 \%{ }^{(2)}$. En América, hallazgos de investigaciones en la misma población evidenciaron porcentajes similares ${ }^{(6-12)}$.

Un estudio realizado en el 2015 para estimar los costos empresariales, es decir el impacto económico, de la violencia contra las mujeres en 25 empresas medianas y grandes en Paraguay, encontró que en el último año, 30,2\% del personal estuvo involucrado en situaciones de violencia contra la mujer como agredida o como agresor, con un promedio de 24,6 ataques violentos al año. Se estimó una pérdida de fuerza laboral por año de 72.337 colaboradores/as y un costo económico equivalente al 2,39\% del PIB de Paraguay, estimado en 734.871.583 USD ${ }^{(13)}$.

El mantenimiento del patriarcado es la teoría más corriente que explica el ejercicio o el padecimiento de la violencia de pareja o la violencia sexual. Esta teoría basada en el dominio masculino en la sociedad con sus normas patriarcales reflejan la desigualdad y la desigualdad de género en la esfera social ${ }^{(3,14,15)}$, legitimando la violencia infligida por la pareja y la violencia sexual cometida por hombres ${ }^{(3)}$.

Entre los factores de riesgo comunitarios y sociales asociados a la victimización de las mujeres, la bibliografía ha citado las normas sociales de género no equitativas ${ }^{(2)}$. También se señala una asociación entre la frecuencia del maltrato a la esposa con la ausencia de participación en grupos de trabajo constituidos exclusivamente por mujeres, lo cual deja establecido que mayores espacios de grupos de trabajo femenino constituirían una protección contra la violencia sufrida por mujeres de parte de su pareja, ya que los mismos proporcionan una fuente estable de apoyo social e independencia de sus esposos y familias (3). Otras investigaciones han señalado el hecho de que pocas mujeres tienen un alto grado de autonomía, lo que constituye un factor que predispone a ser víctima ${ }^{(2,3)}$. 
Tomando en consideración lo precedente y que hoy día se reconoce cada vez más la salud mental de las mujeres como el campo de mayor impacto en el bienestar individual, familiar y social, este estudio se propuso echar luz sobre el rol que desempeña la participación de mujeres en organizaciones sociales y redes familiares con respecto a la condición de ser víctimas de violencia ${ }^{(16)}$, porque la evidencia científica sobre este rol aportará a la prevención de la violencia doméstica.

\section{MATERIALES Y METODOS}

Se realizó una investigación de tipo descriptivo con componentes analíticos, de corte transverso y de carácter cuantitativo con muestreo no probabilístico. De forma retrospectiva se tomó una base de datos de 1046 mujeres que habían acudido a su control prenatal en forma consecutiva a cuatro centros asistenciales públicos del Departamento de Alto Paraná.

Se utilizó un instrumento estructurado conteniendo todas las variables a ser observadas, que fue completado en entrevistas por estudiantes del último año de la carrera de Psicología. Las variables sociodemográficas incluyeron edad, sexo, nivel educativo, procedencia y estado civil.

Para determinar los antecedentes de violencia intrafamiliar, se utilizó la clasificación de UNICEF para violencia doméstica contra mujeres y niñas ${ }^{(7)}$ que define la violencia física como "malos tratos físicos tales como bofetadas, golpes, torsión de brazos, puñaladas, estrangulación, quemaduras, sofocación, patadas, amenazas con armas u otros objetos, y en casos extremos el asesinato"; violencia psicológica o emocional, como "intimidar, atormentar a la víctima y asume diferentes formas: amenazas de abandono o abuso, reclusión en el hogar, vigilancia estricta, amenazas de destitución del cuidado de los hijos, destrucción de objetos, aislamiento, agresiones verbales y humillaciones constantes"; abuso sexual que se define como "relaciones sexuales forzadas, impuestas mediante amenazas e intimidaciones o con la fuerza física, la coerción a prácticas sexuales indeseadas, o la constricción a tener relaciones sexuales con terceros"; ${ }^{(7)}$ negligencia o abandono como "falta de protección y cuidados físicos al niño de parte de sus figuras de custodia, así como el abandono de sus necesidades evolutivas por falta de estimulación cognitivas que no se deben a carencias extremas de recursos socioeconómicos". También se utilizó la escala de Gelles y Strauss que mide desde el uso de la razón, hasta el uso de un arma para resolver conflictos ${ }^{(17)}$.

El cuestionario incluyó además un listado de con 15 preguntas cerradas, pre codificadas referentes a la participación de las embarazadas en organizaciones sociales y redes familiares.

Los datos fueron cargados en una hoja de cálculo Excel y posteriormente analizados en el paquete estadístico SPSS. Los resultados se expresaron en forma de frecuencia absoluta y relativa y para indagar posibles asociaciones entre tipo de violencia y participación en organizaciones sociales y redes familiares género se utilizó la prueba de chi cuadrado a un nivel de significancia de 0,05.

Se respetó la confidencialidad de la información asignando códigos a las encuestas. La participación de las mujeres y de los/las estudiantes fue en forma voluntaria, proporcionándoles información suficiente y clara sobre los objetivos del estudio, y la utilización de los datos investigación antes del llenado del cuestionario que fue de forma anónima y se realizó en el local de las Facultades seleccionadas. Al término de las encuestas, se proporcionó material educativo con respecto a temas de violencia doméstica y salud sexual y reproductiva.

\section{RESULTADOS}

Fueron incluidas en el estudio 1046 gestantes, de las cuales el 60\% tenía 25 o menos años de edad, el 83,2\% vivía en pareja, 12,7\% tenía primaria incompleta o sin educación formal (Tabla 1 ). 
Tabla 1. Características sociodemográficas de las gestantes. $n=1046$

\begin{tabular}{lcc}
\hline Características Sociodemográficas & $\mathbf{n}$ & $\%$ \\
\hline Edad (años) & & \\
$<18$ años & 120 & 11,4 \\
18 a 25 & 508 & 48,4 \\
26 a 35 & 315 & 30 \\
$>35$ & 106 & 10,1 \\
Estado civil & & \\
Conviviendo & 476 & 45,4 \\
Casada & 397 & 37,8 \\
Soltera & 160 & 15,3 \\
Separada & 16 & 1,5 \\
Nivel educativo & & \\
Sin educación formal & 21 & 2 \\
Primaria incompleta & 131 & 12,5 \\
Primaria completa & 295 & 28,1 \\
Secundaria incompleta & 360 & 34,3 \\
Secundaria completa & 133 & 12,7 \\
Universitaria incompleta & 30 & 2,9 \\
Universitaria completa & 14 & 1,3 \\
Técnico & 50 & 5,7 \\
\hline Sin información & & \\
\hline & & \\
\hline
\end{tabular}

En relación al tipo de violencia que las gestantes eran víctimas, 70,1\% fue de violencia psicológica, $24 \%$ de física, $8 \%$ económica y $8 \%$ abuso sexual Tabla 2. 
Tabla 2. Tipos de violencia $n=1046$

\begin{tabular}{lll}
\hline Tipos de violencia & $\mathrm{n}$ & $\%$ \\
\hline Violencia Psicológica & 733 & 70,1 \\
Violencia Física & 250 & 23,9 \\
Abuso sexual & 78 & 7,5 \\
\hline
\end{tabular}

La participación en grupo de iglesia fue la más frecuente con el $41 \%$, seguido de grupo vecinal y cooperadora escolar (Tabla 3).

Tabla 3. Participación en organizaciones. $n=1046$

\begin{tabular}{lcc}
\hline Participación en organizaciones & $\mathbf{n}$ & $\mathbf{\%}$ \\
\hline Grupo de iglesia & 429 & 41,0 \\
Vecinal & 171 & 16,3 \\
Cooperadora escolar & 134 & 12,8 \\
Club deportivo & 58 & 5,5 \\
Organización de mujeres & 36 & 3,4 \\
Sindical & 27 & 2,6 \\
\hline
\end{tabular}

La frecuencia del contacto con los familiares se muestra en la Tabla 4, siendo la visita de madre las más frecuente $(81,4 \%)$, seguida de visita de hermanos.

Tabla 4. Contacto con los familiares. $n=1046$

\begin{tabular}{lllll}
\hline Contacto con los familiares & \multicolumn{2}{c}{ Si } & \multicolumn{2}{c}{ No } \\
& n & \% & n & \% \\
\hline Visita madre & 851 & 81,4 & 195 & 18,6 \\
Visita hermanos & 837 & 80,0 & 209 & 20,0 \\
Visita padre & 774 & 74,0 & 272 & 26,0 \\
Visita amigas & 709 & 67,8 & 337 & 32,2 \\
Visita suegros & 639 & 61,1 & 407 & 38,9 \\
Visita pareja de amigos & 515 & 49,2 & 531 & 50,8 \\
Visita parientes & 507 & 48,5 & 539 & 51,5 \\
\hline
\end{tabular}

Con respecto a la relación entre ser víctima de violencia participación en organizaciones y tener contacto con familiares y amigos, se encontró que le hecho de ser víctima de violencia psicológica estuvo asociado con no participar en cooperadora escolar $(p<0,045)$ y no recibir visita de amigas $(p<0,02)$ (Tabla 5$)$. 
Tabla 5. Asociación entre ser vvíctima de violencia psicológica y participación en organizaciones y contacto con familiares y amigos. $n=1046$

\begin{tabular}{llll}
\hline Víctima de & & $\begin{array}{l}\text { No tiene participación en } \\
\text { cooperadora escolar* }\end{array}$ & $\begin{array}{l}\text { No recibe visita de } \\
\text { amigas** }\end{array}$ \\
violencia & $S i(n=733)$ & $649(88,5 \%)$ & $117(37,40 \%)$ \\
psicológica & No $(n=313)$ & $263(84,0 \%)$ & $220(30,00 \%)$ \\
\hline *Valor de $\mathbf{P}=0,045 ; * *$ Valor $\mathrm{p}=0,02$ &
\end{tabular}

Tanto el hecho de ser víctima de abuso sexual $(p<0,004)$ como ser víctima de violencia económica $(0,028)$ se asociaron con no frecuentar a los padres (Tabla 6$)$.

Tabla 6. Asociación entre ser Víctima de abuso sexual o de violencia económica y participación en eventos sociales. $n=1046$

\begin{tabular}{llll}
\hline Víctima de.... & & $\begin{array}{l}\text { No recibe visita de los } \\
\text { padres }\end{array}$ & Valor de P \\
\hline Abuso sexual & Si $(n=78)$ & $31(39,7 \%)$ & 0,004 \\
& No $(n=968)$ & $241(24,9 \%)$ & \\
Violencia económica & Si $(n=83)$ & $30(36,1 \%)$ & 0,028 \\
& No $(n=963)$ & $242(25,1 \%)$ & \\
\hline
\end{tabular}

\section{DISCUSION}

Las mujeres del estudio tenían características sociales que delatan condiciones de vulnerabilidad. La mayoría era menor de 25 años, cerca de la mitad con educación primaria (completa e incompleta) y en estado de embarazo, condiciones de la esfera individual caracterizadas como de riesgo para ser blanco de violencia doméstica ${ }^{(3)}$. Similares hallazgos se pudieron encontrar en algunos estudios que revelaron cómo las mujeres con un mayor grado de instrucción, escolaridad secundaria o superior, presentaban una probabilidad de $20 \%$ a $55 \%$ menor de ser víctimas de violencia de pareja o violencia sexual que las mujeres con menos educación ${ }^{(20-22)}$.

Las mujeres del presente estudio provenían de áreas urbana y rural, acudían a centros públicos y habían sido víctimas en alto porcentaje de violencia doméstica, que, según se constató, ésta, estaba asociada a su no participación en organizaciones sociales y redes familiares. El aislamiento del entorno social inmediato y de las redes familiares en el que se encuentran mujeres víctimas de violencia psicológica, económica y abuso sexual principalmente ha sido descrito en la bibliografía ${ }^{(2,3)}$ como situaciones que influyen en las opciones de las víctimas de denunciar la situación ${ }^{(18)}$. La condición de víctima de abuso sexual en especial que se constató en este grupo estuvo asociado a no frecuentar a padres y no participar en clubes de madres; Es decir, las víctimas no tenían interacciones con entornos comunitarios, familiares, como padres, madres y hermanos en general y del social inmediato, como ser el ámbito educativo de hijos e hijas u otros, donde se encuentra la posibilidad de que las víctimas expresen o visualicen su condición. Misma situación respecto a la condición de padecer violencia económica y no frecuentar a los padres. Otra investigación dio cuenta de algunos factores que pueden disminuir o amortiguar el riesgo de violencia de pareja como: haber gozado de una crianza sana durante la niñez protege contra la violencia infligida por la pareja y la violencia sexual; contar con una familia propia que ofrece respaldo; vivir dentro de una estructura familiar nuclear o extensa; pertenecer a una asociación; y la capacidad de las mujeres de reconocer el riesgo al que está expuesta ${ }^{(3)}$.

Como factor protector a ser víctima de violencia psicológica se halló el frecuentar a las amigas. Participar en mayor cantidad de espacios de grupos de trabajo femenino serían una protección contra la violencia sufrida por mujeres de parte de su pareja lo cual queda constatado en el reporte de esta investigación. Se requiere por tanto enfatizar en los programas de prevención y eliminación de toda forma de violencia doméstica, la importancia de que mujeres en general frecuenten amigas como una práctica saludable de la vida cotidiana ${ }^{(3)}$. 
La tendencia a asociación significativa que se evidenció entre ser víctima de violencia física y no visitar a padres y madres, replica hallazgos similares de estudios que se han realizado sobre el entorno de la mujer maltratada o abusada en España y Ecuador. En el estudio en 7898 mujeres españolas que presentaron mayor vulnerabilidad a la violencia en la pareja, principalmente inmigrantes, se describió que tenían un nivel socioeconómico bajo, también aumento de la magnitud de esta en aquellas cuya madre fue víctima de violencia de parte de su pareja y que carecían de apoyo social ${ }^{(18)}$. En el segundo estudio, se constató la importancia del entorno comunitario y de las interacciones de víctima y agresor con la familia, vecindario y agentes de intervención; el papel de la familia en relación con el apoyo que brinda a la víctima es en ocasiones ambivalente y puede llegar a ser culpabilizador. La influencia del vecindario y de las redes informales también ha sido significativa ${ }^{(19)}$.

La no asociación entre padecimientos de violencias con no participar en el ámbito sindical, no participar en organizaciones vecinales, cubes de madres clubes deportivos ni organización de mujeres. Así mismo no se asoció a participación en grupos de iglesias y a cooperadora escolar. Respecto a las redes familiares, no se encontró asociación entre ser víctima de violencia psicológica y visitar al padre, a la madre, a parejas de amigos, parientes y suegros.

Una limitación de la investigación se circunscribe a su característica de temporalidad, ya que es retrospectiva, de manera que la información no aporta datos de la realidad actual, que puede haber modificado en las cifras halladas. Otra limitación del estudio constituye el hecho de no haberse realizado con un muestreo probabilístico, lo que limita la capacidad del mismo de convertirse en representativo de la población de un Departamento.

La evidencia científica sobre la temática de prevención de la violencia infligida por la pareja y la violencia sexual se encuentra aún en sus inicios y queda mucho aún por hacer. Se requiere realizar estudios que aporten información sobre la prevalencia y los impactos que tiene en la salud de la mujer, la violencia sexual de pareja íntima en entornos donde hasta el momento no se han realizado, principalmente en los Centros de Atención Prenatal.

\section{REFERENCIAS BIBLIOGRÁFICAS}

1. Organización Mundial de la Salud [Internet]. Ginebra-Suiza: OMS; [citado 12 de agosto de 2013]. Disponible en: http://www.who.int/mediacentre/factsheets/ fs239/es/

2. Organización Mundial de la Salud-Oficina Regional para las Américas-Organización Panamericana de la Salud [Internet]. Washington D.C., EEUU: OPS-OMS; [citado 2 abril 2014]. Disponible en: http://apps.who.int/iris/bitstream/10665/98 816/1/WHO_RHR_12.36_spa.pdf?ua =1

3. Organización Mundial de la Salud-Escuela de Higiene y Medicina Tropical de Londres. [Internet]. Washington D.C.: OPS-OMS; [citado 10 Mayo 2014]. Disponible en:http://apps.who.int/iris/bitstream/10665/ 44810/1/9789275316351_spa.pdf

4. Naciones Unidas [Internet]. Washington, DC: EEUU: Secretario General de Naciones Unidas. [citado 8 de junio 2013]. Disponible en Pg. Web: http://www.un.org/womenwatch/daw/vaw/p ublications/Spanish\%20study.pdf

5. Psiquiatría integral. Servicios en Psiquiatría y Salud Emocional. [Internet]. Buenos AiresArgentina. Rojtenberg S. Mujer: vulnerabilidad, depresión y ansiedad. [citado 2 abril 2016]. Disponible en: http://psiquiatriaintegral.com.mx/principal/? $p=335$

6. Galeano A, Orrego B, Pérez $M$, Ruiz V, Arrom MA, Ruoti $M$, et al. Violencia intrafamiliar sufrida por mujeres en un Hospital Público.
Revista Paraguaya de Psiquiatría. 2015; 3(1): 42-9.

7. Arrom C, Ruoti $M$, Samudio $M$, Orué $E$. Condiciones socioeconómicas de adolescentes embarazadas con síntomas de depresión, consumo de sustancias y víctimas de violencia intrafamiliar. Revista Paraguaya de Psiquiatría. 2015; 3(1):13-9.

8. Arrom C, Ruoti M, Samudio M, Orué E, Arrom CM. Sintomatología depresiva en embarazadas víctimas de abuso sexual. Mem Inst Investig Cienc Salud 2015;13(3):82-7.

9. Arrom C, Samudio M, Arrom CM, Lampert N, Arrom MA, Orué E. Violencia intrafamiliar, de pareja íntima y abuso sexual en puerperio inmediato. Su relación con depresión. Revista Paraguaya de Psiquiatría. 2013; 1(2): 17-23.

10. Ferrari Audi CA, Segall-Corrêa AM, Santiago SM, Andrade MDG, Pérez Escamila R. Violencia doméstica na gravidez: prevalencia e fatores associados. Rev. Saúde Pública 2008; 42(5):877-85.

11. Castro R, Ruíz A. Prevalencia y severidad de la violencia contra mujeres embarazadas, México. Rev Saúde Pública 2004;38(1):62-70.

12. Friedemann-Sánchez G, Lovatón R. Intimate Partner Violence in Colombia: Who Is at Risk?. University of Minnesota. 2012; Social Forces 91(2):663-8.

13. Brendel C, Heikel MA. Los costos empresariales de la violencia contra las mujeres en Paraguay. Cooperación 
Alemana-GIZ. [Internet] [citado 16 de setiembre de 2015]. Disponible en PDF: http://info.comvomujer.org.pe/catalogoco mvo/productoscatalogos_3_2015/ComVoM ujer_ResumendelestudiodecostosPY_2015. pdfCEDAW

14. Arrom CM, Fresco MP, Arrom MA, Arrom C, Ruoti $M$, Orué $E$, et al. Afirmaciones $y$ creencias sobre violencia de género y autonomía de la mujer en población consultante. Estudio comparativo. Revista Científica Estudios e Investigaciones, UNIBE. 2015; 4(1): 9-20.

15. González Vera M. Patriarcado, machismo e impunidad: causas de la cotidiana violencia de género En: Coordinadora de Derechos Humanos del Paraguay (Codehupy). Derechos humanos en Paraguay/ Yvypóra Derécho Paraguáipe. Codehupy. Asunción: Mercurio S.A.; 2012:447-63.

16. Montero I, Aparicio D, Gómez-Beneytoc B, Moreno-Küstnerd $B R$, Usallf J, VázquezBarquerog JL et al. Género y salud mental en un mundo cambiante. Gac Sanit 2004; 18 (4): 175-81.

17. Gelles R, Straus M. Intimate Violence. The definitive study of the causes and consequences of abuse in the american family. Published by the Simon \& Schuster Trade Division. Manufactured in the United States of America, 1988.

18. Sanz-Barbero B, Rey L, Otero-García L. Estado de salud y violencia contra la mujer en la pareja. Gac Sanit [Internet]. 2014 Abr citado 2016 Nov 11]; 28(2):102$8 . \quad$ Disponible en: http://scielo.isciii.es/scielo.php?script=sci arttext\&pid =S0213-

91112014002200003\&lng=es. http://dx.d oi.org/10.1016/j.gaceta.2013.08.004.

19. Boira S, Carbajosa P, Méndez R. Miedo, conformidad y silencio. La violencia en las relaciones de pareja en áreas rurales de Ecuador. Psychosocial Intervention 2016: 25: 9-17. Disponible en: http://eds.a.ebscohost.com/eds/pdfviewer/ pdfviewer?vid $=19 \&$ sid $=804756 \mathrm{~d} 2$-93af$464 \mathrm{e}-\mathrm{a} 5 \mathrm{a} 2-$ f40f376cdcbb\%40sessionmgr4009\&hid $=42$ 02

20. Brown L, Thurman T, Bloem J, Kendall C. Sexual violence in Lesotho. Studies in Family Planning, 2006; 37(4):269-80.

21. Fehringer $J A$, Hindin MJ. Like parent, like child: Intergenerational transmission of partner violence in Cebu, the Philippines. Journal of Adolescent Health, 2009; 44(4):363-71.

22. Flake DF. Individual, family, and community risk markers for domestic violence in Peru. Violence Against Women 2005; 11(3):353-73.

23. Herrmann MG, Van Klaveren A. Disminución de la participación de la población en organizaciones sociales durante los últimos trece años en Chile e implicaciones para la construcción de una política de planificación urbana más participativa. EURE 2016; 42(125): 175203. 